\title{
Research on Symbol Synchronization Algorithm of LTE System
}

\author{
Wenjing Zhang \\ Chongqing Communication Institute of PLA \\ Chongqing, China \\ e-mail: zhang_zhou2010@yahoo.cn
}

\author{
Baoqin Wang and Xu Fang and Xuyang Zhou and \\ Weichao Sha \\ Chongqing Communication Institute of PLA and \\ Chongqing Communication Institute of PLA and \\ Logistical Engineering University of PLA and \\ Chongqing Communication Institute of PLA \\ Chongqing, China and Chongqing, China \\ and Chongqing, China and Chongqing, China \\ e-mail: dyzhuo@yahoo.com.cn and \\ fangxu_1985@163.com and smthroot@gmail.com and \\ ahnust@163.com
}

\begin{abstract}
This paper designs and realizes algorithm of symbol synchronization for LTE system. In order to validate the feasibility of symbol timing synchronization algorithm, a series of simulations are performed with LTE channel models and the simulation results are analyzed carefully. Simulation shows that this algorithm has a great advantage of system implementation. It can be found the symbol start position within the shortest time.
\end{abstract}

Keywords-LTE; synchronization signal; symbol timing synchronization; simulation

\section{INTRODUCTION}

LTE which is regarded as the "4G" technology has brought great impact to the mobile communication. It has improved and enhanced 3G air access technology. It uses OFDM and MIMO as its wireless network evolution on the basis of technology. This technology can enhance the capacity for sustained development of the mobile communications industry. But there exists a synchronization problem in the LTE system. Synchronization is very important in telecommunication system, synchronization error will cause inter-symbol interference (ISI) and intercarrier interference (ICI), and affect the system performance badly. Symbol timing synchronization is a rough estimate of the starting position of the FFT window. It can help to demodulate FFT correctly [1].

Symbol timing synchronization is one of the synchronization problems that LTE system needs to solve. Because the exact timing of the signal delay and the transmission signal to the receiver is unknown, the receiver does not know the correct starting position of the OFDM symbol [2]. Symbol timing synchronization is to find the correct starting position of OFDM symbol. Due to the presence of the cyclic prefix, LTE system on the symbol synchronization requirements is not as stringent as other communication systems. As long as the starting point for the FFT window falls into the range of cyclic prefix, the receiver can demodulate received data correctly and does not cause intersymbol interference.

Symbol timing synchronization algorithms can be broadly divided into the following two categories: One is based on the aided algorithm of training sequence data (dataaided), that is, the sending end insert some known pilot frequency information in the transmitted data frame. The receiving end and the known signal correlation operation can complete synchronization. This algorithm is also known as the synchronization algorithm based on pilot frequency [4] [5].This algorithm obtains a better synchronization performance, but it increases the cost of the system overhead. It is generally used in the case of bursty data services. It can quickly achieve symbol timing synchronization and can improve the synchronization efficiency. But it increases the system overhead and reduces the bandwidth efficiency. The second is use the redundancy data of the received signal (usually use the information of cyclic prefix) to the estimated blind estimation algorithm (the non-data-aided). Therefore, it is also known as synchronization algorithm which is based on CP [6] [7]. But the intersymbol interference will effect on this kind of algorithm and it is estimated that it has performance deterioration in the multipath channel environment.

In the process of establishing synchronization, symbol timing synchronization is usually first. Only to determine the exact starting position of the OFDM symbol, the FFT will be demodulated correctly. From analysing of the LTE system physical layer frame structure, we know that the period of primary synchronization signal is $5 \mathrm{~ms}$, that is, each half frame contains an identical master synchronization signal. The master synchronization signal is generated by the ZC sequence. After the IFFI transformation it becomes the time domain signal, and then it will be transmitted over wireless channel. The length of the master synchronization signal frequency domain is 62 points, After modulating OFDM, it will be transmitted in system minimum bandwidth. The length of the time domain is 128 points. The symbol timing synchronization generally is completed in the time domain. The master synchronization signal has a good correlation in the time domain, by correlation process of time domain will be able to obtain timing synchronization information. 


\section{SYMBOL TIMING SYNCHRONIZATION ALGORITHM}

\section{A. Algorithm Description}

If there are three different local master synchronization signals, and the receiver does not know which main synchronization signal the sender will send, then the local synchronization signal needs to be processed three times. It should be found that the period of primary synchronization signal is 5ms. Study of LTE frame structure can be found that the primary synchronization signal has the periodicity of $5 \mathrm{~ms}$, that is two half frames of a radio frame to transmit the same primary synchronization signal, using the LTE system in the primary synchronization signal which has a $5 \mathrm{~ms}$ cycle time domain repeatability, using the time domain of a predetermined step size to slide and cross-correlation, and realizing estimate of downlink timing rough synchronization.

If strong interference service sub-frame exists during the communication process, conventional time domain sliding correlation process can't avoid that correlation value of the position of the strong interference is stronger than the correlation value of the primary synchronization position. It will lead to timing synchronization error and subsequent frequency synchronization failure. Using normalized time domain processing can effectively resist the strong interference in the system timing synchronization performance. In this paper, synchronization scheme uses segmentation strategy to resist that strong initial frequency offset influence on relevant performance.

If receiving a wireless frame data, the two half frames for a predetermined length of the slide step sliding correlation operation, we will get the estimation of the measurement matrix:

$$
\begin{aligned}
& \operatorname{corr}(\mathrm{d})=\sum_{\mathrm{n}=0}^{\mathrm{L}-1} \mathrm{r}^{*}\left(\mathrm{n}+\mathrm{d}^{*} \text { step }\right) \mathrm{r}\left(\mathrm{n}+\mathrm{N} / 2+\mathrm{d}^{*} \text { step }\right) \\
& \operatorname{pow}(d)=\sum_{n=0}^{N / 2-1} r^{*}(n+N / 2+d) r(n+N / 2+d) \\
& M(d)=\frac{\mid \text { corr }\left.(d)\right|^{2}}{(\text { pow }(d))^{2}} \\
& \hat{\mathrm{d}}=\underset{\mathrm{d}}{\arg \max }\{\mathrm{M}(\mathrm{d}) \mid 0 \leq \mathrm{d} \leq \mathrm{N} /(2 * \text { step })-1\}
\end{aligned}
$$

In this formula, $\mathrm{N}$ represents the length of the received wireless frame data. $\mathrm{L}$ represents the length of the correlation sequence. pow $(d)$ represents the average energy of received half-frame length signal. $\mathrm{M}(\mathrm{d})$ represents metric function, and $\mathrm{M}(\mathrm{d})$ is the maximum, the maximum value on the corresponding position is the starting position $\hat{\mathrm{d}}$ of the primary synchronization signal.

\section{B. Simulation Platform}

This simulation platform is suitable for algorithm simulation of LTE system receiver symbol timing synchronization. Because it considers the baseband digital signal simulation only, does not take code into account. The simulation link simplifies the actual sending and receiving process of the LTE system. Simulation tools use the Matlab7.0. Signal transmitter and receiver block diagram of the simulation platform is shown in Figure 1.

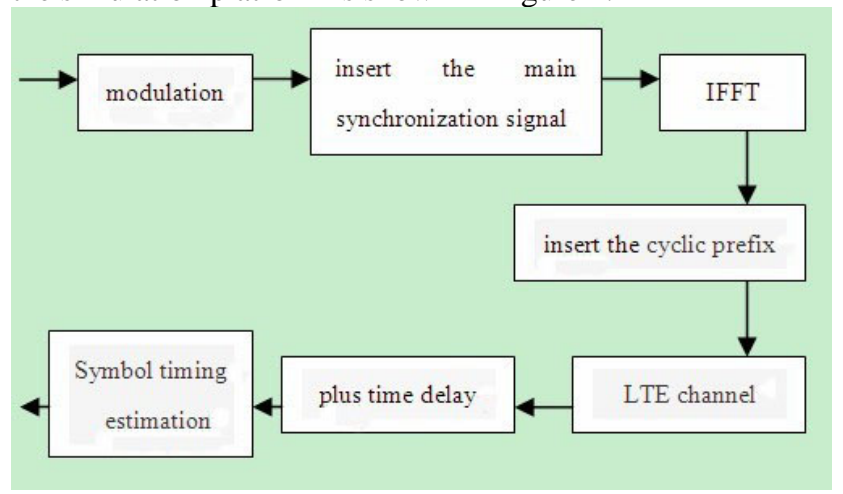

Figure 1. Simulation platform

First, modulate the input data stream, insert the primary synchronization signal in accordance with the predetermined rule of the LTE system the primary synchronization signal is inserted, then transform domain signal into time domain signal by using the transformation of IFFT. After adding cyclic prefix, it will become the data stream of one radio frame in the LTE system. Add the delay value before passing the channel. The channel uses the model of the regulation LTE system.

\section{Simulation Parameters}

TABLE I. SYMBOL TIMING SYNCHRONIZATION SIMULATION PARAMETERS TABLE

\begin{tabular}{|l|l|}
\hline \multicolumn{1}{|c|}{ Name } & \multicolumn{1}{c|}{ Directions } \\
\hline channel bandwidth & $20 \mathrm{MHz}$ \\
\hline signal bandwidth & $18 \mathrm{MHz}$ \\
\hline radio frame length & $10 \mathrm{~ms}$ \\
\hline subframe length & $1 \mathrm{~ms}$ \\
\hline timeslot length & $0.5 \mathrm{~ms}$ \\
\hline cyclic prefix type & normal cyclic prefix \\
\hline cyclic prefix length & $144 / 160$ \\
\hline $\begin{array}{l}\text { master synchronization } \\
\text { signal }\end{array}$ & $\begin{array}{l}\text { primary sync signal is inserted } \\
\text { in the LTE protocol }\end{array}$ \\
\hline subcarrier interval & $15 \mathrm{KHz}$ \\
\hline timeslot number of symbols & $7 \mathrm{OFDM} \mathrm{symbols}$ \\
\hline FFT points & 2048 \\
\hline modulation mode & QPSK \\
\hline sampling frequency & $30.72 \mathrm{MHz}$ \\
\hline carrier frequency & $2.3 \mathrm{GHz}$ \\
\hline
\end{tabular}




\section{Simulation Conditions}

(1) Initial frequency offset is in the range of $[-13 \mathrm{KHz}$, $13 \mathrm{KHz}]$;

(2) Master synchronization signal is generated randomly;

(3) Amplitude and phase is imbalances: the amplitude is imbalance about degree $0 \mathrm{~dB}$ and the phase is imbalance in 5 degrees, the initial phase is set to 0 ;

(4) Number of segments is $M=8$;

(5) Sliding step is step $=16$;

(6) Channel environment: EPA of 3GPP TS 36.101 (R8)

EPA stipulated (maximum Doppler shift $5 \mathrm{~Hz}$ );

(7) Test SNR point: 0dB,-10dB;

\section{E. Simulation Results and Analysis}

This emulation will verify the performance of the scheme form the different signal to noise ratio and different initial frequency offset. This scheme uses a predetermined sliding step correlation to compute. The expected timing of the synchronization accuracy is \pm 8 Ts.

1) Influence of signal to noise ratio

The signal to noise ratio influences on the performance of synchronization:

According to the above simulation parameters of the design: If the sampling rate of receiving data is $30.72 \mathrm{MHz}$, the correct starting position of the master synchronization signal is located at 36265 .First, this simulation will receive the data sampling down to $1.92 \mathrm{MHz}$, so the correct starting position of the master synchronization signal is 2205. Simulation is carried out in accordance with the above simulation parameters.

The following figure is the curve of $0 \mathrm{~dB}$ and-10dB SNR single a simulation synchronization. From the Fig. 2 we can see when the SNR is 0dB, the peak is 26.2182 and the peak appears in the 139th window. The peak is 8.7329 when the SNR is $-10 \mathrm{~dB}$, correlation peak decreases, but the peak also appeared in the 139th window. Because the sliding step length is 16 , we can estimate the location of the master synchronization signal, it is $(139-1) * 16+1=2209$. It meets the expected timing synchronization requirements. The simulations show that the synchronization program still has a good anti-noise ability.
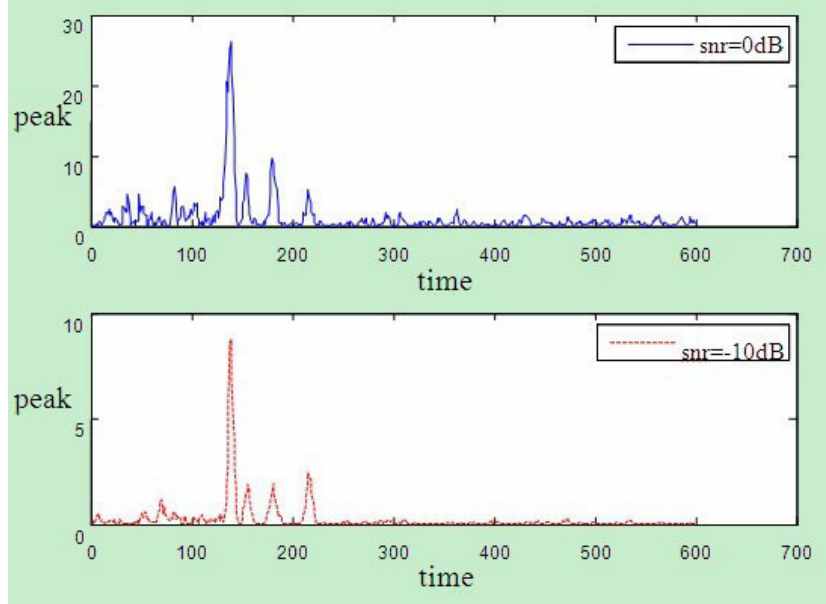

Figure 2. SNRof $-10 \mathrm{~dB}$ and $0 \mathrm{~dB}$ symbol timing synchronization algorithm simulation curve

\section{2) Influence of initial frequency offset}

Initial frequency offset influences on the synchronization performance:

According to the above simulation parameters we can infer, if the sampling rate of received data is $30.72 \mathrm{MHz}$, then correct starting position of the master synchronization signal is located in 36265. Now simulation will receive the data sampling down to $1.92 \mathrm{MHz}$ first, the correct starting position of the master synchronization signal is 2205, signal to noise ratio is $10 \mathrm{~dB}$, we use the above simulation parameters to simulate.

Figure 3 is synchronization curve of single simulation when the initial frequency offset are $1 \mathrm{KHz}$ and $10 \mathrm{KHz}$. From the Figure 3 we can see when initial frequency offset is $1 \mathrm{KHz}$, correlation peak value appears in the 139th window, the peak is 12.2504. Correlation peak value also appears in the 139th window when initial frequency offset is $10 \mathrm{KHz}$. The peak is 8.1555 . So the peak is decreased. The result shows that this program still has a good ability to withstand strong initial frequency.

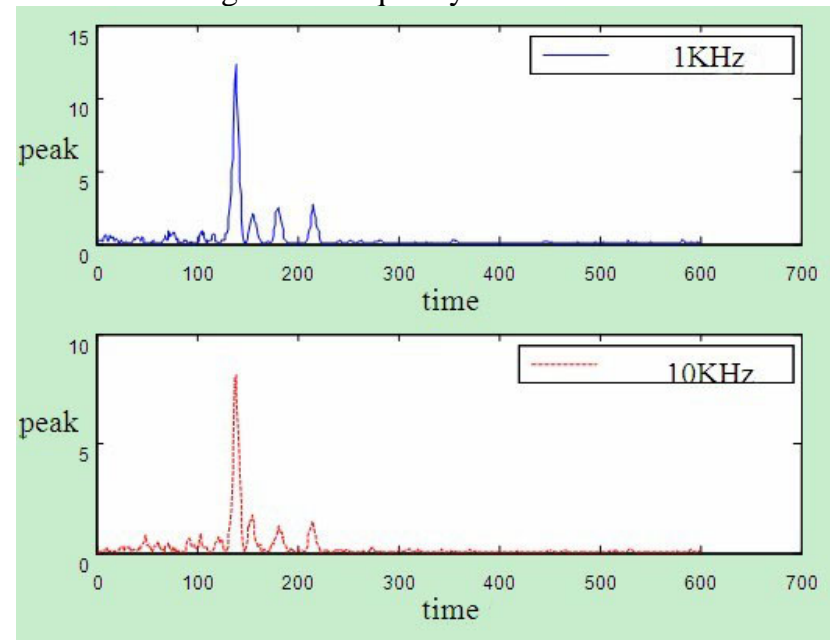

Figure 3. Symbol synchronization algorithm simulation curve of initial frequency for $1 \mathrm{KHz}$ and $10 \mathrm{KHz}$

\section{F. Algorithm Complexity}

The following will calculate complexity of symbol timing synchronization scheme from the complex multiplication and plural plus the number of times. To assume that the LTE system in a half frame data length is 9600 samples, the master synchronization signal is 128 sample points, if the sliding step is set to 16 , the algorithm to calculate the amount for the $9600 / 16 \times 128$ complex multiply accumulate.

Figure 4 is the simulation of different slip step glide $=[16$ 24 32] synchronization algorithm complexity. With the sliding step increasing, the amount of calculation for this synchronization program will be decreased. 


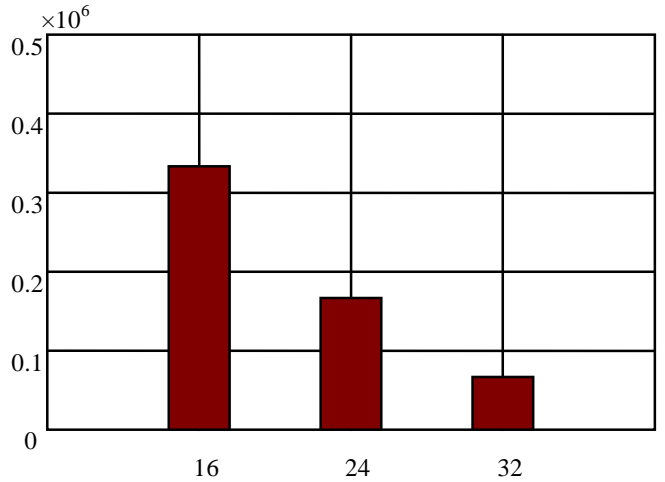

Figure 4. Algorithm complexity of symbol timing synchronization

\section{SUMMARIES}

This paper studies symbol timing synchronization algorithm of the LTE system and verifies the feasibility of the algorithm. Simulation analysis shows that symbol synchronization algorithm has a great advantage of system software implementation. It is able to quickly locate in the shortest possible time.

\section{REFERENCES}

[1] B.P.Lathi, Modern Digital and Analog Communication Systems, Third ed., Oxford Univ.press,1998.

[2] Ch. Nanda Kishore and V. Umapathi Reddy. A frame synchronization and frequency offset estimation algorithm for OFDM system and its analysis. EURASIP Joural on Wireless Communications and Networking,vol.2006.

[3] 3GPP TR 25.913 V7.3.0 Requirements for Evolved UTRA(E-UTRA) and Evolved UTRAN(E-UTRA). Article in a conference proceedings:

[4] F Classen, H Meyr. Frequency synchronization algorithms for OFDM Systems suitable for communication over frequency-selective fading channels. in Prco.IEEE vehic.Technol.Conf., Stockholm. Swedem, 1994(3):1655-1654.

[5] Schmidl T M, cox D c. Robust frequency and timing synchronization for OFDM.IEEE Transactions Communications,1997,45(12):1616-1621.

[6] Roh H, Chenu K, Park J. An MMSE fine carrier frequency synchronization algorithm for OFDM systems[J]. IEEE Trans. on Consumer Electronics, 1997, 43:761-766.

[7] Helard J. Time synchronization without specific symbols for OFDM. IEEE Electronic letters, 1999,35(2):130-132. 